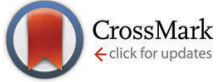

Cite this: Phys. Chem. Chem. Phys., 2015, 17, 15756

Received 27th March 2015, Accepted 6th May 2015

DOI: $10.1039 / c 5 c p 01805 f$

www.rsc.org/pccp

\title{
An important impact of the molecule-electrode coupling asymmetry on the efficiency of bias- driven redox processes in molecular junctions
}

\begin{abstract}
Ioan Bâldea†
Two recent experimental and theoretical studies (Proc. Natl. Acad. Sci. U. S. A., 2014, 111, 1282-1287; Phys. Chem. Chem. Phys., 2014, 16, 25942-25949) have addressed the problem of tuning the molecular charge and vibrational properties of single molecules embedded in nanojunctions. These are molecular characteristics escaping so far from an efficient experimental control in broad ranges. Here, we present a general argument demonstrating why, out of various experimental platforms possible, those wherein active molecules are asymmetrically coupled to electrodes are to be preferred to those symmetrically coupled for achieving a(n almost) complete redox process, and why an electrochemical environment has advantages over "dry" setups. This study aims at helping to nanofabricate molecular junctions using the most appropriate platforms allowing the broadest possible bias-driven control over the redox state and vibrational modes of single molecules linked to electrodes.
\end{abstract}

\section{Introduction}

In spite of impressive advances in nanoelectronics, ${ }^{1-4}$ detailed characterization and control of molecular properties under in situ conditions continue to remain important challenges for fabricating and understanding single-molecule junctions. To this aim, more recent studies have emphasized the need to go beyond electronic transport. ${ }^{5-7}$ Vibrational properties studied via inelastic electron tunneling spectroscopy (IETS) ${ }^{8}$ and surface enhanced Raman spectroscopy (SERS) ${ }^{5,6,9-12}$ may represent such a valuable piece of information, ideally if they are acquired concomitantly using transport measurements. This has been demonstrated in a recent joint experimental theoretical study on fullerene-based electromigrated junctions ${ }^{13}$ and a theoretical study $^{14}$ on viologen-based junctions in the electrochemical (EC) scanning tunneling microscope (STM) setup. The latter was based on experimental data reported previously. ${ }^{15}$

Ref. 13 and 14 indicated that a significant tuning of vibrational frequencies and Raman scattering intensities can be obtained via bias-driven changes in the charge of the active molecule in a current carrying state; tuning the molecular charge via applied biases allows us to control chemical bond strengths and, thence, vibrational properties.

Obviously, the broadest control that can be achieved pursuing this route corresponds to the complete change in the average

Theoretische Chemie, Universität Heidelberg, Im Neuenheimer Feld 229, D-69120 Heidelberg, Germany. E-mail: ioan.baldea@pci.uni-heidelberg.de $\dagger$ National Institute for Lasers, Plasmas, and Radiation Physics, Institute of Space Sciences, Bucharest, Romania. redox state of the molecule by adding an entire electron. To be specific, we limit ourselves to n-type (LUMO-mediated) conduction (orbital energy offset $\varepsilon_{0}>0$ ), as this is the case for the molecular junctions reported in ref. 13, 14 and 16. Reaching this ideal limit was impossible in the experiments reported in ref. 13. On the other hand, ref. 14 indicated that an almost perfect reduced $(n \approx 1)$ state can be reached in electrolytically gated junctions. ${ }^{15}$

Are the obstacles to obtaining a full reduction in some molecular junctions merely of technical nature? This is the question that initiated the present study. By comparing performances of various nanofabrication platforms and identifying the ones (depicted in Fig. 5a and 6a below) that are most advantageous for achieving an almost complete reduction, the present study aims at helping to design molecular junctions allowing the broadest bias-driven control over the molecular charge and vibrational properties.

\section{Model}

The framework adopted here is provided by the single-level Newns-Anderson model, which was discussed ${ }^{17,18}$ and validated for a variety of molecular junctions, ${ }^{19-21}$ including those used in the existing SERS-transport studies. ${ }^{13,14}$ We checked that reorganization effects $^{14,17,18,22-24}$ do not qualitatively change the present conclusions. This is illustrated by the comparison between Fig. 3 and 4, Fig. 1 and 2, and between panels $b$ and $c$ of Fig. 6 . To make the presentation as simple 

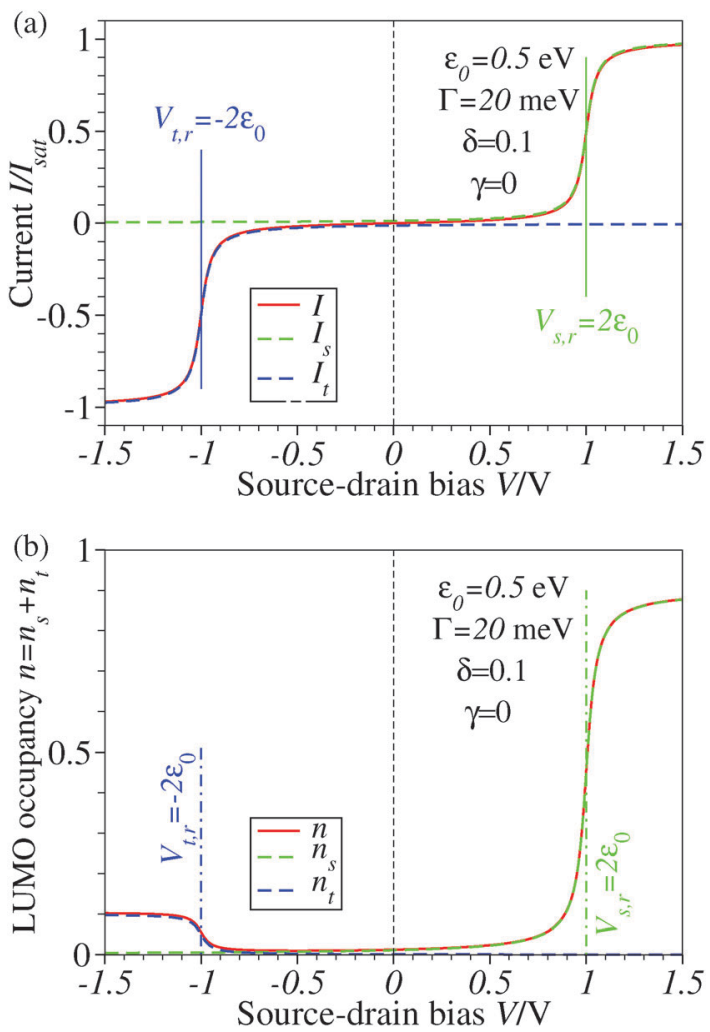

Fig. 1 Results for $\varepsilon_{0}=0.5 \mathrm{eV}, \Gamma=20 \mathrm{meV}, \delta=0.1$, and $\gamma=0$ showing that the biases $V$ where steps and plateaus in the current $I$ and LUMO occupancy $n$ occur are controlled by the values $V_{\mathrm{s}, \mathrm{r}}$ and $V_{\mathrm{t}, \mathrm{r}}$ (see eqn (5) and (6) of the main text) where the LUMO becomes resonant with the Fermi levels of electrodes s(ubstrate) and t(ip), respectively. Note that the reduction efficiency (quantified by the LUMO occupancy $n$ ) is strongly dependent on the bias polarity in the case of an asymmetric molecule electrode coupling $\left(\Gamma_{\mathrm{s}} \neq \Gamma_{\mathrm{t}}\right)$, unlike the current plateau value, which is unchanged upon bias polarity reversal. $n_{\mathrm{s}}$ and $n_{\mathrm{t}}$ represent the separate electrodes' contributions to the LUMO occupancy $n$ expressed by the first and second terms in the RHS of eqn (5) from the main text. For better readability, a vertical dashed line marks the zero bias reference.

and clear as possible, we will give below only formulae wherein reorganization effects are disregarded; details of the ensemble averaging needed to include these effects are not given here but can be found elsewhere. ${ }^{17,18,24}$

Eqn (1), (5), (3) and (4), ${ }^{17,18,24}$ which allow us to express the current $I$ and the LUMO occupancy $n$, constitute the framework of the present discussion ( $e$ and $h$ are the elementary charge and Planck's constant, respectively).

$$
\begin{gathered}
I=\frac{I_{\mathrm{sat}}}{\pi}\left[\arctan \frac{\varepsilon_{0}(V)+\frac{e V}{2}}{\Gamma}-\arctan \frac{\varepsilon_{0}(V)-\frac{e V}{2}}{\Gamma}\right] \\
I_{\mathrm{sat}}=2 \pi e \Gamma_{\mathrm{s}} \Gamma_{\mathrm{t}} /(h \Gamma) \\
\varepsilon_{0}(V)=\varepsilon_{0}+\gamma e V \\
\Gamma_{\mathrm{s}}=2 \Gamma(1-\delta) ; \Gamma_{\mathrm{t}}=2 \Gamma \delta
\end{gathered}
$$
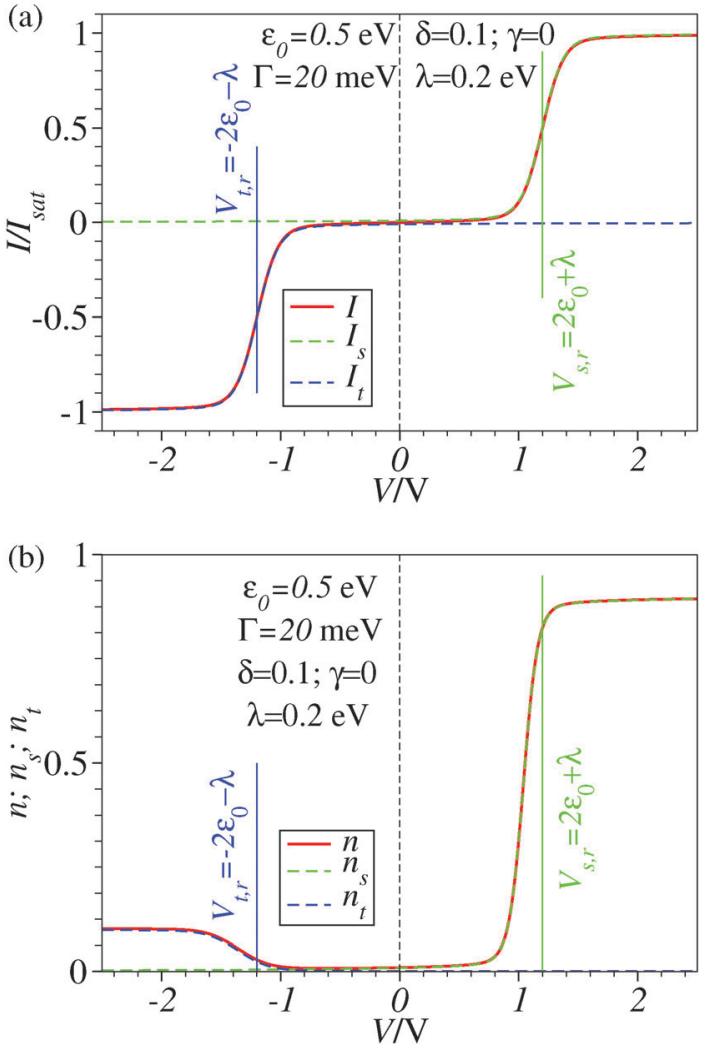

Fig. 2 Results similar to those of Fig. 1 including reorganization effects quantified by the reorganization energy $\lambda$ whose value is given in the legend. For better readability, a vertical dashed line marks the zero bias reference.

The LUMO occupancy $n$ of the molecule embedded in a biased junction comprises contributions $n_{\mathrm{s}, \mathrm{t}}$ from the two ( $\mathrm{s}$ and $\mathrm{t}$ ) electrodes ${ }^{25,26}$

$$
\begin{gathered}
n=n_{\mathrm{s}}+n_{\mathrm{t}} \\
n_{\mathrm{s}}=\frac{1-\delta}{\pi} \operatorname{arccot} \frac{\varepsilon_{0}(V)-e V / 2}{\Gamma} \\
n_{\mathrm{t}}=\frac{\delta}{\pi} \operatorname{arccot} \frac{\varepsilon_{0}(V)+e V / 2}{\Gamma}
\end{gathered}
$$

The Newns-Anderson model embodies two asymmetries characterized by the dimensionless parameters $\gamma$ and $\delta$ defined in eqn (3) and (4). The potential profile asymmetry (or voltage division factor) $\gamma$ quantifies the bias-induced shift in the LUMO energy offset relative to the Fermi energy $\left(E_{\mathrm{F}} \equiv 0\right)$ with respect to the case of unbiased electrodes $\varepsilon_{0} \rightarrow \varepsilon_{0}(V) \cdot{ }^{18,19,27} \Gamma_{\mathrm{s}, \mathrm{t}}$ denote the couplings of the molecule to generic "left"/"substrate" (s) and "right"/"tip" ( $t$ ) electrodes. Without loss of generality (because it merely amounts to appropriately label the electrodes) we assume that s(ubstrate) is not the weakest coupled electrode to the LUMO $\left(\Gamma_{\mathrm{t}} \leq \Gamma_{\mathrm{s}}, 0<\delta \leq 1 / 2\right)$ and define the source-drain bias $V$ with respect to its potential $\left(V=\mathscr{V}_{\mathrm{t}}-\mathscr{V}_{\mathrm{s}}\right)$. Using a potential origin located symmetrically between the electrodes' Fermi energies $\left(-e^{\mathscr{V}_{\mathrm{s}, \mathrm{t}}}= \pm e V / 2\right),-1 / 2<\gamma<1 / 2 . \gamma$ is positive (negative) if the bias shifts the LUMO towards the Fermi level of electrode $s(t)$. 
Similar to $I$ (Fig. 1 and 2) ${ }^{14}$ the LUMO occupancy exhibits substantial changes within ranges $\delta V \sim \Gamma$ (which are narrow in typical non-resonant cases $\Gamma \ll \varepsilon_{0}$ ) at the biases (case $\lambda=0$ )

$$
\begin{gathered}
V_{\mathrm{r}, \mathrm{s}}=\varepsilon_{0} /(1 / 2-\gamma) \\
V_{\mathrm{r}, \mathrm{t}}=-\varepsilon_{0} /(1 / 2+\gamma)
\end{gathered}
$$

where the LUMO and electrodes' Fermi level become resonant and plateaus (saturation) above these biases. The saturation values (subscript sat) can be expressed as follows (superscripts \pm refer to bias polarity)

$$
\begin{gathered}
n \underset{\text { large positive } V}{\stackrel{V-V_{\mathrm{r}, ~} \gg \Gamma}{\longrightarrow}} n_{\text {sat }}^{+} \approx 1-\delta \\
n \underset{\text { large negative } V}{\stackrel{\left|V-V_{\mathrm{r}, t}\right| \gg \Gamma}{\longrightarrow}} n_{\text {sat }}^{-} \approx \delta
\end{gathered}
$$

\section{Results and discussion}

The impact of the source-drain bias on the molecular charge is depicted in Fig. 3 and 4. It is worth noting that the asymmetry in electronic coupling $\left(\delta \neq 1 / 2, \Gamma_{\mathrm{s}} \neq \Gamma_{\mathrm{t}}\right)$ is physically distinct from the asymmetry $(\gamma \neq 0)$ in the electric potential drop across a molecular junction. ${ }^{14,19,28}$ Their impact on $n$ is completely different. The comparison between Fig. $3 \mathrm{a}$ and $\mathrm{b}$ reveals that $\gamma$ $\neq 0$ merely breaks the bias reversal symmetry $(n(V) \neq n(-V))$. This is the counterpart of current rectification $(I(-V) \neq-I(V)$, $c f$. eqn (1) and (3)). As long as the molecule-electrode coupling is symmetric $(\delta=1 / 2)$, the plateau values at either bias polarity are equal, $n_{\mathrm{sat}}^{+}=n_{\mathrm{sat}}^{-}=1 / 2$.

From the perspective of achieving a full redox process in biased molecular junctions, this result for symmetric coupling is disappointing: whatever high is the source-drain bias which a molecular junction can withstand $\left(|V| \gg V_{\mathrm{r}, \mathrm{s}}\right.$ or $\left.\left|V_{\mathrm{r}, \mathrm{t}}\right|\right)$, whether the potential profile is symmetric $(\gamma=0)$ or asymmetric $(\gamma \neq 0)$, the average molecular charge cannot exceed half of an electron $\left(n_{\text {sat }}^{ \pm}=1 / 2\right.$ for $\delta=1 / 2, c f$. eqn (5)). This is a surprising result; intuitively, one may expect a full change in the molecular redox state $(n=1)$ at biases corresponding to resonant transport $\left(\varepsilon_{0}(V) \approx \mu_{\mathrm{s}}(V)\right.$ or $\left.\mu_{\mathrm{t}}(V)\right) .{ }^{13}$ In fact, exactly on resonance $\left(V=V_{\mathrm{r}, \mathrm{s}}\right.$ or $V_{\mathrm{r}, \mathrm{t}}$ ) the average molecular charge is even smaller: $n \approx 1 / 4$, see eqn (12) and (13) for $\delta=1 / 2, \Gamma \ll \varepsilon_{0}$.

$$
\begin{aligned}
& n\left(V=V_{\mathrm{r}, \mathrm{s}}\right)=\underbrace{\frac{1-\delta}{2}}_{n_{\mathrm{s}}}+\underbrace{\frac{\delta}{\pi} \arctan \frac{\Gamma}{e V_{\mathrm{r}, \mathrm{s}}}}_{n_{\mathrm{t}}} \stackrel{\Gamma \ll \varepsilon_{0}}{\longrightarrow} \frac{1-\delta}{2} \\
& n\left(V=V_{\mathrm{r}, \mathrm{t}}\right)=\underbrace{\frac{1-\delta}{\pi} \arctan \frac{\Gamma}{e\left|V_{\mathrm{r}, \mathrm{t}}\right|}}_{n_{\mathrm{s}}}+\underbrace{\frac{\delta}{2}}_{n_{\mathrm{t}}} \stackrel{\Gamma \ll \varepsilon_{0}}{\longrightarrow} \frac{\delta}{2}
\end{aligned}
$$

According to eqn (10) and (11), a molecule in a current-carrying state can accommodate an average charge larger than $n=1 / 2$ for junctions characterized by asymmetric molecule-electrode couplings $\delta \neq 1 / 2$. The stronger the asymmetry, the larger is the
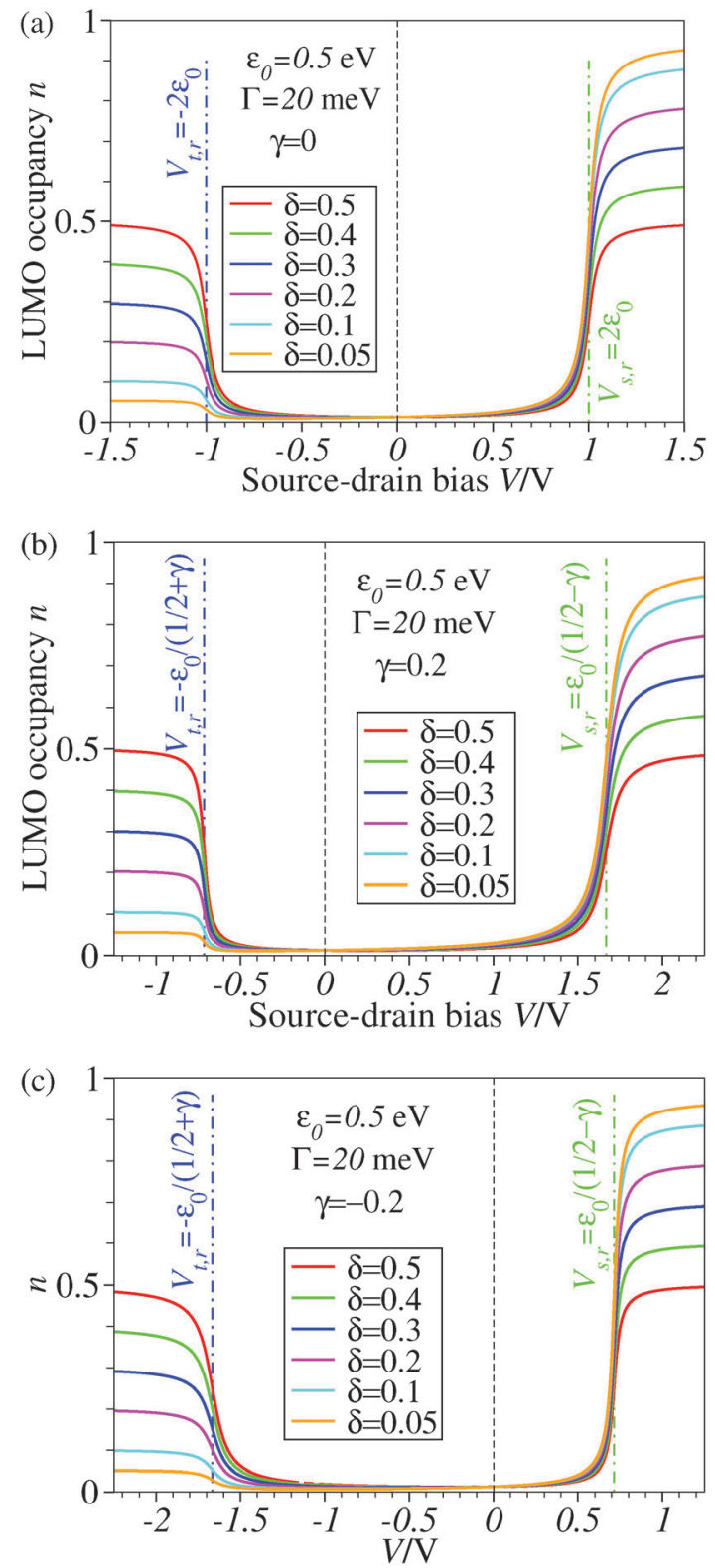

Fig. 3 The stronger the asymmetry of molecule-electrode couplings (smaller $\delta^{\prime} s$ ), the more efficient is the LUMO reduction. Note that a nonvanishing value of $\gamma$ (panels $b$ and $c$ ) breaks the bias reversal symmetry $(n(-V) \neq n(V)$ but does not change the plateau values of $n$ with respect to the case $\gamma=0$ (panel a). The values of $\varepsilon_{0}$ and $\Gamma$ are comparable to those used in existing concurrent SERS-transport studies. ${ }^{13,14}$ For better readability, a vertical dashed line marks the zero bias reference.

charge of the average redox state (Fig. 3). An almost perfect reduced state $(n \approx 1)$ can be achieved for highly asymmetric moleculeelectrode couplings $(\delta \ll 1)$ if the potential of the strongest coupled electrode (s) is sufficiently negative (sufficiently large positive biases). In such cases $\left(\Gamma_{\mathrm{s}} \gg \Gamma_{\mathrm{t}}\right.$ and $\left.V-V_{\mathrm{r}, \mathrm{s}} \gg \Gamma / e\right)$, the Fermi energy of electrode $\mathrm{s}$ is sufficiently above the LUMO energy, and the electron transferred from this electrode at a high rate $\left(\Gamma_{\mathrm{s}}\right)$ almost entirely remains on the LUMO in cases where the transfer from the LUMO to electrode $\mathrm{t}$ is inefficient (small $\left.\Gamma_{\mathrm{t}}\right): n \approx \Gamma_{\mathrm{s}} /\left(\Gamma_{\mathrm{s}}+\Gamma_{\mathrm{t}}\right)=$ $1-\delta \lesssim 1$ (Fig. 5a). This is impossible in cases of symmetric 

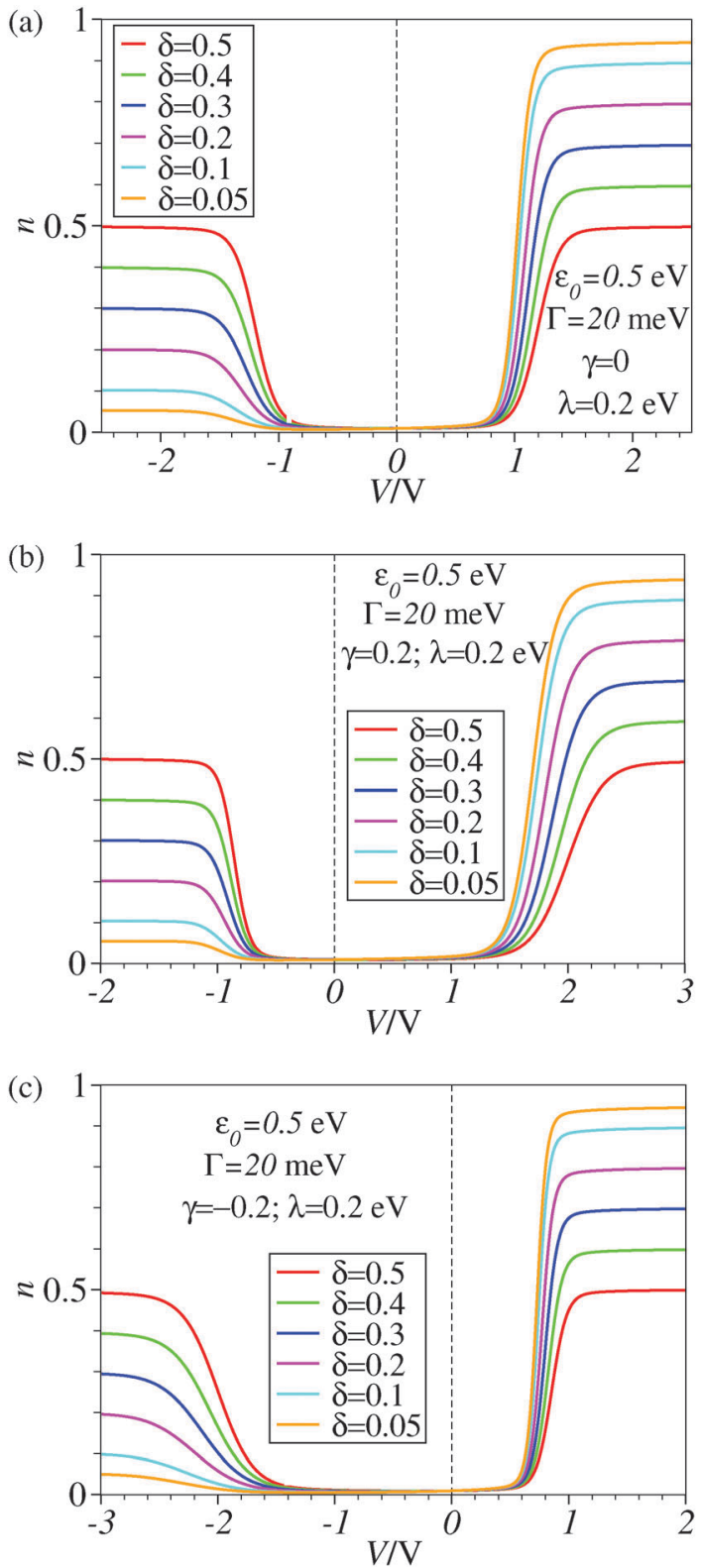

Fig. 4 Results similar to those of Fig. 3 including reorganization effects quantified by the reorganization energy $\lambda$ whose value is given in the legend. For better readability, a vertical dashed line marks the zero bias reference.

coupling $\left(\Gamma_{\mathrm{s}}=\Gamma_{\mathrm{t}}\right)$, wherein $n \approx \Gamma_{\mathrm{s}} /\left(\Gamma_{\mathrm{s}}+\Gamma_{\mathrm{t}}\right)=1 / 2$; on average, half of the entire electron transferred from electrode $\mathrm{s}$ to the LUMO is further transferred to electrode $t$ in cases wherein the two transfer rates are equal (Fig. 5 b).

The foregoing analysis demonstrated that an almost complete reduction can be obtained (i) for a molecule highly asymmetrically coupled to electrodes and (ii) for sufficiently high biases. While the first condition $\left(\Gamma_{\mathrm{t}} \ll \Gamma_{\mathrm{s}}\right)$ can be easily satisfied in asymmetrical EC-STM (see Section 4.2$)^{14,26}$ setups, the second condition seems problematic. Fig. 1 illustrates that the biases needed for an almost perfect reduction are those where current plateaus occur.
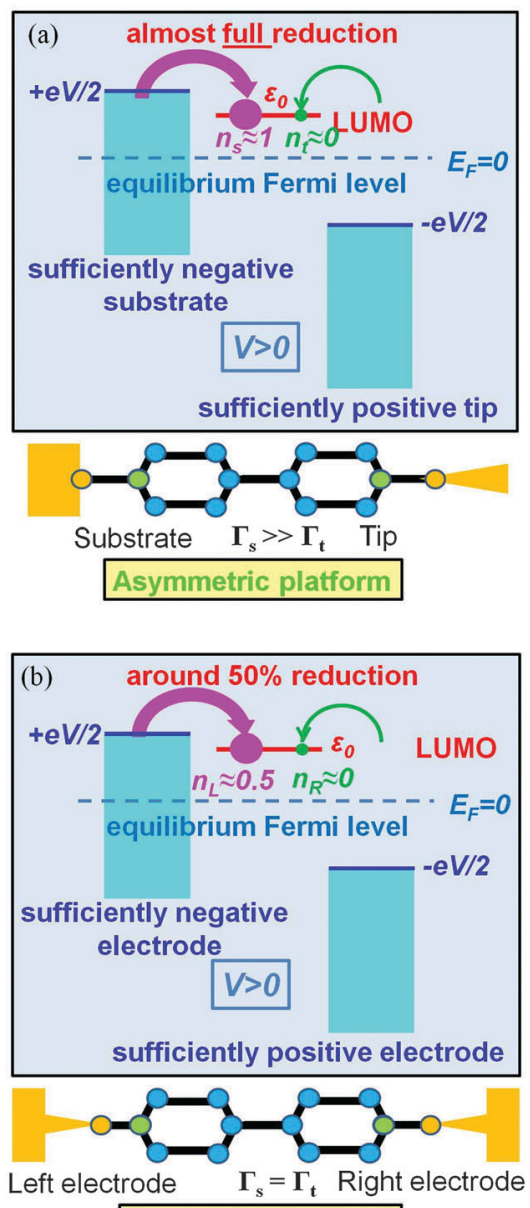

Symmetric platform

Fig. 5 An almost complete reduction can be achieved if the LUMO is much stronger coupled to one electrode $\left(\Gamma_{\mathrm{S}} \gg \Gamma_{\mathrm{t}}\right)$ having a sufficiently negative potential (panel a), which is impossible for symmetric coupling to electrodes (panel b).

As experiments on molecular junctions did not routinely report currents exhibiting plateaus with increasing bias (we are aware of one exception ${ }^{29}$ ), this appears to be an important practical limitation.

A nearly complete reduction would be possible if the LUMO lies below the Fermi level of the strongest coupled electrode $(n \approx 1-\delta$ for $\delta \ll 1$ form eqn (5)) or below the Fermi levels of both electrodes (Fig. 6). This results from eqn $(5): n \approx(1-\delta)+$ $\delta=1$ for $\varepsilon_{0}<0$ (the LUMO below the Fermi level $E_{\mathrm{F}}(=0)$ of unbiased electrodes). In the absence of any bias, $\varepsilon_{0}>0$; the Fermi level lies within the HOMO-LUMO gap of a molecule linked to electrodes (charge neutrality). However, $\varepsilon_{0}<0$ becomes possible during electrostatic gating..$^{15,30-32}$ An appropriate gate potential $V_{\mathrm{G}}$ (overpotential in the electrochemical language, on which the LUMO energy $\varepsilon_{0}$ linearly depends ${ }^{15,25,26,31}$ ) can lower the LUMO energy below $E_{\mathrm{F}}$ (Fig. 6a). $I-V_{\mathrm{G}}\left(I-\varepsilon_{0}\right)$ transfer characteristics exhibiting maxima, which occur at resonance $\left(\varepsilon_{0} \approx 0\right),{ }^{15,18,24}$ can be taken as an indication of a substantial change in the molecular redox state (from $n \approx 0$ for $\varepsilon_{0}>0$ to $n \approx 1$ for $\varepsilon_{0}<0$ ). This is illustrated in Fig. $6 \mathrm{~b}$, which 

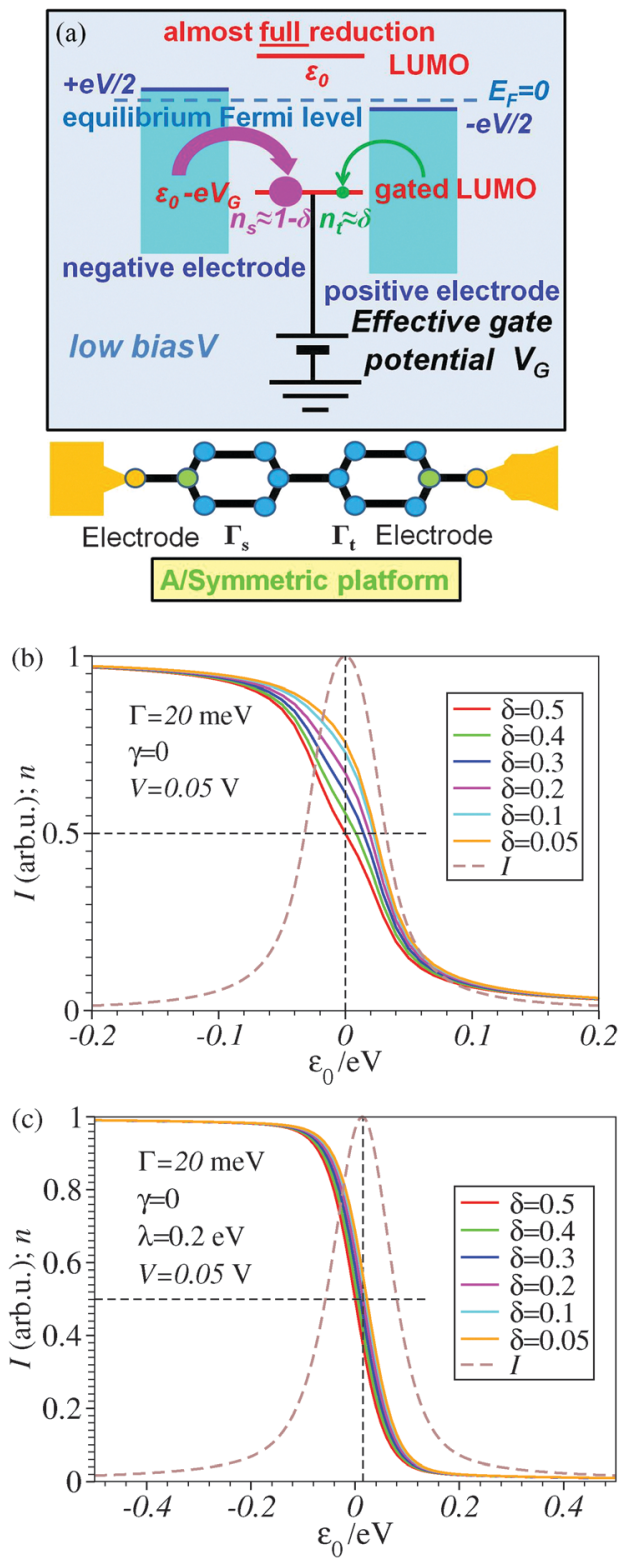

Fig. 6 Results demonstrating that an efficient reduction can be achieved by lowering the LUMO energy below the electrodes' Fermi level via LUMO gating (panel a). An almost complete reduction (values of $n$ close to unity) can be obtained for reasonable gate-driven LUMO shifts; in this case, the coupling asymmetry $\delta$ plays a secondary role: $n \approx 95 \%$ (almost $\delta$-independent) at $\varepsilon_{0} \approx$ $-0.1 \mathrm{eV}$ (without reorganization effects: panel b; with reorganization effects: panel $c$ ). These results emphasize that it is not the (source-drain) bias $V$, but rather the overpotential (on which $\varepsilon_{0}$ linearly depends) that determines the LUMO occupancy $n$ in the electrochemical environment.

emphasizes that it is not the (source-drain) bias $V$, but rather the overpotential that determines the reduction efficiency in the electrochemical environment.

\section{Specific remarks}

In this section we will address three specific issues related to the results reported above.

\subsection{Rectification $v s$. reduction}

To avoid possible misunderstandings, in this subsection we briefly discuss some of the present results in the context of those reported previously.

In agreement with previous work (e.g., ref. 33 and 34), eqn (1)-(3) show that asymmetric molecule-electrode couplings alone (i.e., $\Gamma_{\mathrm{s}} \neq \Gamma_{\mathrm{t}}($ or $\delta \neq 1 / 2)$ and $\gamma=0$ ) do not yield rectification: the aforementioned equations yield $I(-V)=$ $-I(V)$ for $\gamma=0$ irrespective of the value of $\delta$.

The fact that a potential profile asymmetry $(\gamma \neq 0)$ yields current rectification has been amply discussed. A very incomplete list includes ref. 19, 29, 34 and 35; note that the parameters $p$ of ref. 29 and 35 and $a$ of ref. 34 correspond to that denoted by $\gamma$ in this paper. In particular, the present eqn (1) coincides with eqn (4b) of ref. 34 . This is why all conclusions on the current asymmetry ("current rectification") emerging from our eqn (1) are not new; they coincides with those of the earlier studies based on the same formula for the current $I=I(V)$.

Important new results reported in the present paper are that an applied bias can yield an asymmetric molecular reduction $n(-V) \neq n(V)$ and that current asymmetry $I(-V) \neq-I(V)$ and reduction asymmetry $n(-V) \neq n(V)$ are conceptually different. In particular, a symmetric current $I(-V)=-I(V)$ (no rectification) does not rule out an asymmetric LUMO occupancy $n(-V) \neq n(V)$ (cf. Fig. 1) and current rectification $I(-V) \neq-I(V)$ does not rule out symmetric plateau values of the reduction degree $\left(n\left(V \ll-2 \varepsilon_{0}\right)=n\left(V \gg 2 \varepsilon_{0}\right)=1 / 2\right.$ for $\left.\Gamma_{\mathrm{s}}=\Gamma_{\mathrm{t}}\right)$.

Concerning the current rectification a final comment is in order, however. Similar to ref. 33 and 34, eqn (1)-(3) refer to situations wherein reorganization effects are ignored (i.e., $\lambda \equiv 0$ ). As recent work demonstrated, ${ }^{26}$ in cases where reorganization effects are non-negligible $(\lambda \neq 0)$, the asymmetry $\Gamma_{\mathrm{s}} \neq \Gamma_{\mathrm{t}}$ yields current rectification $(I(-V) \neq-I(-V))$ even if there is no bias-induced shift of the LUMO energy $(\gamma=0)$; see eqn (13), (18) to (23) of ref. 26 and 36 Without intending to be exhaustive (current rectification in not our primary aim here), we mention that, in cases wherein $\Gamma_{\mathrm{s}} \neq \Gamma_{\mathrm{t}}$ and $\gamma=0$, current rectification may also appear due to charging effects. ${ }^{37,38}$

\subsection{Symmetric vs. asymmetric contact couplings and experimental platforms}

The above analysis demonstrated the key role played by the molecule-electrode couplings $\left(\Gamma_{\mathrm{x}}, \mathrm{x}=\mathrm{s}, \mathrm{t}\right)$ in determining the reduction efficiency. For electrodes with wide flat band structures around the Fermi energy, $\Gamma_{\mathrm{s}, \mathrm{t}}$ can be expressed in terms of the electrode density of states at the Fermi level $\rho_{\mathrm{s}, \mathrm{t}}$ and effective transfer integrals $t_{\mathrm{s}, \mathrm{t}}$ quantifying the charge transfer between electrodes and the dominant molecular orbital (LUMO in the specific case considered here) $)^{39}$

$$
\Gamma_{\mathrm{x}}=\rho_{\mathrm{x}} t_{\mathrm{x}}^{2}
$$

Let us now consider the experiment that succeeded to reveal a change in the redox state of a $\mathrm{C}_{60}$ molecule embedded in a biased junction by means of simultaneous SERS-transport measurements in an electromigration platform. ${ }^{13}$ In view of 
the high molecular symmetry and of the (nearly) symmetric electrodes characterizing electromigrated junctions, one can assume $\rho_{\mathrm{s}} \approx \rho_{\mathrm{t}}$ and $t_{\mathrm{s}} \approx t_{\mathrm{t}}$. This implies $\Gamma_{\mathrm{s}} \approx \Gamma_{\mathrm{t}}$, and we have shown above that in this symmetric case $(\delta=1 / 2)$ reduction cannot exceed 50\%. Drawing (experimentalists') attention on the limited reduction degree that can be achieved even if such electromigrated molecular junctions can be brought into a current plateaus regime represents an important aim of the present theoretical work. ${ }^{40}$

In typical STM or CP-AFM experiments, molecules forming regular self-assembled monolayers (SAMs) are (covalently) bound to the substrate. A difference $\rho_{\mathrm{s}} \neq \rho_{\mathrm{t}}$ (yielding $\Gamma_{\mathrm{s}} \neq \Gamma_{\mathrm{t}}$ via eqn (14)) may exist because the substrate surface is typically monocrystalline (e.g., $\mathrm{Au}(111))$ while the tip facet is undefined. Still, it is more probable that not the difference $\rho_{\mathrm{s}} \neq \rho_{\mathrm{t}}$ but rather the charge transfer efficiency $\left(t_{\mathrm{s}} \neq t_{\mathrm{t}}\right)$ determines the contact coupling asymmetry $\Gamma_{\mathrm{s}} \neq \Gamma_{\mathrm{t}}$.

Quantifying the asymmetry $\Gamma_{\mathrm{s}} \neq \Gamma_{\mathrm{t}}$ from transport measurements is not straightforward; we have seen above that (nearly) symmetric curves $I(-V) \approx-I(V)$, which are measured in numerous junctions based on symmetric (and occasionally also asymmetric) molecules, do not exclude (highly) asymmetric contact couplings.

A coupling asymmetry $\Gamma_{\mathrm{s}}>\Gamma_{\mathrm{t}}$ has been concluded in ref. 41 after a detailed analysis of the transport data in CP-AFM junctions. In CP-AFM setups such an asymmetry appears to be plausible because one can expect that $t_{\mathrm{s}}>t_{\mathrm{t}}$, given the fact that a stable molecule-tip chemical bond is hard to imagine; normally, charge transport only occurs by applying a loading force at the CP-AFM tip. ${ }^{42}$ A similar inequality $\left(t_{\mathrm{s}}>t_{\mathrm{t}}, \Gamma_{\mathrm{s}}>\Gamma_{\mathrm{t}}\right)$ can also be expected for STM break junctions; the formation of a stable molecule-tip chemical bond is implausible during repeated processes of rapidly crashing the tip into and retracting it away from the substrate. ${ }^{43,44}$ So, although per se the asymmetric outlook of the STM and CP-AFM setups does not necessarily imply $\Gamma_{\mathrm{s}}>\Gamma_{\mathrm{t}}$, this inequality can be expected in view of the different bond strength at the contacts. The fact that the symmetry of the electromigration platform and the asymmetry of the STM platform do not merely refer to the usual schematic illustrations of these setups (like those in Fig. 5 and 6), but also have a physical content has been recently quantified. ${ }^{4,46}$

Out of the various experimental platforms employed, the EC-STM setup appears to be the most favorable, allowing almost perfect reduction. In the electrochemical environment (EC-STM) ${ }^{15}$ the tip typically approaches but does not come in contact with the free end of the molecules. The different through-bond $v s$. throughspace charge transfer mechanisms at the (EC-)STM substrate and the tip reflect themselves in significantly different transfer integrals $\left(t_{\mathrm{s}} \gg t_{\mathrm{t}}\right)$ responsible for the high asymmetry $\Gamma_{\mathrm{s}} \gg \Gamma_{\mathrm{t}}$ (cf. eqn (14)); in agreement with this analysis, our recent studies demonstrated very highly asymmetric molecule-electrode couplings: $\delta \sim 10^{-4}$ (ref. 26) and $\delta \sim 10^{-2}$ (ref. 14).

\subsection{The Newns-Anderson model}

As noted in the Introduction section, the present study has been motivated by the findings reported in ref. 13 and 14, which demonstrated that reduction is possible in molecular junctions in current carrying states and concluded that the Newns-Anderson model (single level + Lorentzian transmission) represents an adequate theoretical framework. In favor of the the Newns-Anderson model one can still add its ability to excellently describe the charge transport by tunneling in a variety of molecular junctions. ${ }^{19,20,21,33}$ The aforementioned findings represent a sufficient justification for adopting the Newns-Anderson model in the present study.

Still, for reasons delineated below we believe that our main conclusion on the impact of coupling asymmetry $\left(\Gamma_{\mathrm{s}} \neq \Gamma_{\mathrm{t}}\right)$ on the reduction efficiency holds beyond the Newns-Anderson framework.

Using the expressions of the partial LUMO occupancies $n_{\mathrm{s}}$ and $n_{\mathrm{t}}$ given in eqn (6) and (7), respectively, eqn (1) can be rewritten as follows

$$
\begin{gathered}
I=e\left(\frac{n_{\mathrm{s}}}{\tau_{\mathrm{t}}}-\frac{n_{\mathrm{t}}}{\tau_{\mathrm{s}}}\right) \\
\tau_{\mathrm{s}, \mathrm{t}}=\frac{\hbar}{2 \Gamma_{\mathrm{s}, \mathrm{t}}}
\end{gathered}
$$

In eqn (15) (where the factor 2 is the spin contribution), the first term refers to an electron arriving at the LUMO as a result of the coupling of the molecule to the left electrode. Eqn (6) and (4) yield

$$
n_{\mathrm{s}}=\frac{\Gamma_{\mathrm{s}}}{2 \Gamma} \frac{1}{\pi} \operatorname{arccot} \frac{\varepsilon_{0}(V)-e V / 2}{\Gamma} \propto \Gamma_{\mathrm{s}}
$$

This electron is transferred to the right electrode within a characteristic time $\tau_{\mathrm{t}}$. In eqn (16), $\tau_{\mathrm{t}}$ corresponds to the rate $\Gamma_{\mathrm{t}}$ determined by coupling to the right electrode. Likewise, the second term of eqn (15) describes the electron flow in the opposite direction: an electron arriving at the LUMO as a result of the coupling of the molecule to the right electrode. Eqn (7) and (4) yield

$$
n_{\mathrm{t}}=\frac{\Gamma_{\mathrm{t}}}{2 \Gamma} \frac{1}{\pi} \operatorname{arccot} \frac{\varepsilon_{0}(V)+e V / 2}{\Gamma} \propto \Gamma_{\mathrm{t}}
$$

This electron is transferred to the left electrode within a characteristic time $\tau_{\mathrm{s}}$. In eqn (16), $\tau_{\mathrm{s}}$ corresponds to the rate $\Gamma_{\mathrm{s}}$ determined by coupling to the left electrode.

Eqn (17) and (18) yield the following limiting (plateau) values

$$
\begin{aligned}
& n \underset{\text { large positive } V}{\stackrel{V-V_{\mathrm{r}, \mathrm{s}} \gg \Gamma}{\longrightarrow}} n_{\text {sat }}^{+} \approx n_{\mathrm{s}}^{+} \approx \frac{\Gamma_{\mathrm{s}}}{2 \Gamma} \approx\left\{\begin{array}{l}
\stackrel{\Gamma_{\mathrm{s}} \gg \Gamma_{\mathrm{t}}}{\longrightarrow} 1 \\
\stackrel{\Gamma_{\mathrm{s}} \approx \Gamma_{\mathrm{t}}}{\longrightarrow} \frac{1}{2}
\end{array}\right. \\
& n \underset{\text { large negative } V}{\stackrel{\left|V-V_{\mathrm{r}, \mathrm{t}}\right| \gg \Gamma}{\longrightarrow}} n_{\mathrm{sat}}^{-} \approx n_{\mathrm{t}}^{+} \approx \frac{\Gamma_{\mathrm{t}}}{2 \Gamma} \approx\left\{\begin{array}{l}
\stackrel{\Gamma_{\mathrm{s}} \gg \Gamma_{\mathrm{t}}}{\longrightarrow} 0 \\
\stackrel{\Gamma_{\mathrm{s}} \approx \Gamma_{\mathrm{t}}}{\longrightarrow} \frac{1}{2}
\end{array}\right.
\end{aligned}
$$

It is noteworthy that both the current, eqn (15), and the plateau LUMO occupancies, eqn (17) and (18) can be entirely expressed in terms of the rates $\Gamma_{\mathrm{s}, \mathrm{t}}$. Parenthetically, these equations also hold in the presence of reorganization $(\lambda \neq 0)$. In view of the appealing simplicity of the above expressions and their clear 
physical content, we believe that they apply beyond the NewnsAnderson framework, as also suggested by the cartoons presented in Fig. 5 and 6.

Obviously, the aforementioned findings should not be taken as an attempt to discourage alternative approaches of redox processes in molecular junctions based on other theoretical models utilized in the literature ${ }^{39}$ or by further refinements of the Newns-Anderson model itself. Concerning the latter possibility, an extension that appears to us as particularly important in studying charge transport through redox units is to consider population dependent contact couplings $\Gamma_{\mathrm{s}, \mathrm{t}}=\Gamma_{\mathrm{s}, \mathrm{t}}(n)$ and LUMO energy $\varepsilon_{0}=\varepsilon_{0}(n)$.

From numerous studies on redox electrochemical systems, ${ }^{47}$ it is known that changes in the reduction (or oxidation) degree-with accompanying redistributions of the electronic charge over the whole molecule-have an overall, although selective impact on the chemical bond strengths between various molecular constituents and molecular orbital energies. For molecular junctions, the influence of the reduction (or oxidation) degree on the bond strengths at the contacts is of particular interest; so, the transfer integrals $t_{\mathrm{s}, \mathrm{t}}$ (and thence $\Gamma_{\mathrm{s}, \mathrm{t}}$, $c f$., eqn (14)) are expected to be affected.

\section{Conclusions}

We believe that the new theoretical results reported in the present paper offer relevant information for a better theoretical understanding of the microscopic processes occurring in molecular junctions away from equilibrium and useful hints for future experimental investigation.

We have identified experimental platforms allowing almost complete bias-controlled redox processes in molecular junctions. Information on the bias $\left(V\right.$ and $\left.V_{\mathrm{G}} \sim \varepsilon_{0}\right)$ dependent LUMO (in the specific case examined) occupancy $n$, the quantity on which we have focused our attention, can be obtained from bias dependencies of vibrational frequencies $\omega_{\nu}\left(V, \varepsilon_{0}\right)=[1-$ $\left.n\left(V, \varepsilon_{0}\right)\right] \omega_{\nu}^{\mathrm{n}}+n\left(V, \varepsilon_{0}\right) \omega_{\nu}^{\mathrm{a}}$ and Raman scattering intensities $A_{\nu}\left(V, \varepsilon_{0}\right)=$ $\left[1-n\left(V, \varepsilon_{0}\right)\right] A_{\nu}^{\mathrm{n}}+n\left(V, \varepsilon_{0}\right) A_{\nu}^{\mathrm{a}}$ extracted from simultaneous SERStransport measurements, which take values interpolating between the relevant (neutral $n$ and anionic a) charge species. ${ }^{14}$ We have demonstrated that the experimental setup asymmetry plays an essential role in achieving an almost perfect reduced state via bias tuning; efficient reduction is possible within some molecular electronic platforms, but definitely impossible within other platforms. We have shown that reduction can at most reach $50 \%$ in two-terminal setups wherein a molecule is symmetrically coupled to electrodes (Fig. 5b). In contrast, an almost complete redox process can be obtained in cases of highly asymmetric molecule-electrode couplings (Fig. 5b). On this basis, an improved reduction can be expected in fullerenebased junctions if an STM platform (highly asymmetric coupling) is adopted instead of the electromigration platform (symmetric couplings) utilized in experiments. ${ }^{13}$

Further, we found that an almost full reduction in two-terminal setups is accompanied by current plateaus. Because such plateau effects have been observed in molecular junctions based on the zwitterionic molecule hexadecylquinolinium tricyanoquinodimethanide $\left(\mathrm{C}_{16} \mathrm{H}_{33} \mathrm{Q}_{3}{ }^{-} \mathrm{CNQ}\right)^{29}$ (seemingly the only known example), concurrent SERS-transport measurements ${ }^{13,14}$ on this system could be of interest to investigate bias-driven changes in molecular vibrational properties.

The fact that, unlike existing orbital gating measurements using "dry", platforms, ${ }^{32}$ experiments resorting to electrolyte gating ${ }^{15,30,31}$ succeeded to reveal such a maximum renders the electrochemical three-terminal (EC-STM) platform a promising route in achieving an efficient reduction in molecular electronic devices, ${ }^{14}$ also because it does not require high biases hardly accessible experimentally and the coupling asymmetry is not critical (cf. Fig. 6b).

\section{Acknowledgements}

Financial support provided by the Deutsche Forschungsgemeinschaft (grant BA 1799/2-1) is gratefully acknowledged.

\section{References}

1 S. J. Van der Molen, N. R. Naaman, E. Scheer, J. B. Neaton, A. Nitzan, D. Natelson, N. J. Tao, H. van der Zant, M. Mayor, M. Ruben, M. Reed and M. Calame, Nat. Nanotechnol., 2013, 8, 385-389.

2 M. A. Ratner, Nat. Nanotechnol., 2013, 8, 378-381.

3 S. H. Choi, B. Kim and C. D. Frisbie, Science, 2008, 320, 1482-1486.

4 E. Lörtscher, Nat. Nanotechnol., 2013, 8, 381-384.

5 J.-H. Tian, B. Liu, X. Li, Z.-L. Yang, B. Ren, S.-T. Wu, N. Tao and Z.-Q. Tian, J. Am. Chem. Soc., 2006, 128, 14748-14749.

6 D. R. Ward, D. A. Corley, J. M. Tour and D. Natelson, Nat. Nanotechnol., 2010, 6, 33-38.

7 S. V. Aradhya and L. Venkataraman, Nat. Nanotechnol., 2013, 8, 399-410.

8 B. C. Stipe, M. A. Rezaei and W. Ho, Science, 1998, 280, 1732-1735.

9 L. L. Zhao, L. Jensen and G. C. Schatz, Nano Lett., 2006, 6, 1229-1234.

10 Z. Liu, S.-Y. Ding, Z.-B. Chen, X. Wang, J.-H. Tian, J. R. Anema, X.-S. Zhou, D.-Y. Wu, B.-W. Mao, X. Xu, B. Ren and Z.-Q. Tian, Nat. Commun., 2011, 2, 305.

11 B. Liu, A. Blaszczyk, M. Mayor and T. Wandlowski, ACS Nano, 2011, 5, 5662-5672.

12 T. Konishi, M. Kiguchi, M. Takase, F. Nagasawa, H. Nabika, K. Ikeda, K. Uosaki, K. Ueno, H. Misawa and K. Murakoshi, J. Am. Chem. Soc., 2013, 135, 1009-1014.

13 Y. Li, P. Doak, L. Kronik, J. B. Neaton and D. Natelson, Proc. Natl. Acad. Sci. U. S. A., 2014, 111, 1282-1287.

14 I. Bâldea, Phys. Chem. Chem. Phys., 2014, 16, $25942-25949$.

15 I. V. Pobelov, Z. Li and T. Wandlowski, J. Am. Chem. Soc., 2008, 130, 16045-16054.

16 The discussion in this paper, couched in terms of the LUMO and reduction, refers to molecular junctions exhibiting 
n-type conduction. For molecular junctions with p-type conduction, the following replacements should be done: LUMO $\rightarrow$ HOMO, reduction $\rightarrow$ oxidation, $\varepsilon_{0}=\varepsilon_{\text {LUMO }} \equiv$ $E_{\mathrm{LUMO}}-E_{\mathrm{F}} \rightarrow-\varepsilon_{0}=\varepsilon_{\mathrm{HOMO}} \equiv E_{\mathrm{F}}-E_{\mathrm{HOMO}}>0$ and $\gamma \rightarrow-\gamma$.

17 W. Schmickler, J. Electroanal. Chem., 1986, 204, 31-43.

18 I. G. Medvedev, Phys. Rev. B: Condens. Matter Mater. Phys., 2007, 76, 125312.

19 I. Bâldea, Phys. Rev. B: Condens. Matter Mater. Phys., 2012, 85, 035442.

20 I. Bâldea, J. Am. Chem. Soc., 2012, 134, 7958-7962.

21 (a) I. Bâldea, Nanoscale, 2013, 5, 9222-9230; (b) I. Bâldea, Z. Xie and C. D. Frisbie, Nanoscale, 2015, DOI: 10.1039/c5nr02225h.

22 W. Schmickler and N. Tao, Electrochim. Acta, 1997, 42, 2809-2815.

23 A. N. Kuznetsov and W. Schmickler, Chem. Phys., 2002, 282, 371-377.

24 I. Bâldea, J. Phys. Chem. C, 2014, 118, 8676-8684.

25 I. G. Medvedev, J. Electroanal. Chem., 2007, 600, 151-170.

26 I. Bâldea, J. Phys. Chem. C, 2013, 117, 25798-25804.

27 F. Zahid, M. Paulsson and S. Datta, Electrical Conduction through Molecules, Advanced Semiconductors and Organic Nano-Techniques, Academic Press, 2003, vol. 3.

28 J. Hodgkiss, E. Zysman-Colman, S. Higgins, G. Solomon, I. Bâldea, I. Samuel, L. Venkataraman, F. Wudl, B. Xu, R. Venkatramani, H. Ottosson, D. Perepichka, U. Lemmer, P. Skabara, A. Mount and D. Bradley, Faraday Discuss., 2014, 174, 125-151.

29 R. M. Metzger, T. Xu and I. R. Peterson, J. Phys. Chem. B, 2001, 105, 7280-7290.

30 N. J. Tao, Phys. Rev. Lett., 1996, 76, 4066-4069.

31 A. Alessandrini, S. Corni and P. Facci, Phys. Chem. Chem. Phys., 2006, 8, 4383-4397.

32 H. Song, Y. Kim, Y. H. Jang, H. Jeong, M. A. Reed and T. Lee, Nature, 2009, 462, 1039-1043.

33 I. Bâldea, Chem. Phys., 2012, 400, 65-71.
34 G. Zhang, M. A. Ratner and M. G. Reuter, J. Phys. Chem. C, 2015, 119, 6254-6260.

35 I. R. Peterson, D. Vuillaume and R. M. Metzger, J. Phys. Chem. A, 2001, 105, 4702-4707.

36 For clarity, let us still mention that, although present, current rectification is hardly visible within the drawing accuracy shown in Fig. 2.

37 F. Zahid, A. W. Ghosh, M. Paulsson, E. Polizzi and S. Datta, Phys. Rev. B: Condens. Matter Mater. Phys., 2004, 70, 245317.

38 We note that, in fact, the current rectification driven by the charging effects reported in ref. 37 relies just upon the population asymmetry $n(-V) \neq n(V)$ discussed in the present paper.

39 J. C. Cuevas and E. Scheer, Molecular Electronics: An Introduction to Theory and Experiment, World Scientific Publishers, 2010.

40 For the sake of simplification, the present discussion ignores the fact that the C60 molecule has a threefold degenerate LUMO. The bias range sampled in the experiment is far away from the current plateaus ( $c f$. Fig. S1-S3 of ref. 13), and even multiplied by 3 ; the $n$-values are less than $10 \%$ ( $c f$. Fig. $4 \mathrm{~b}$ and Fig. S7, ESI of ref. 13).

41 X. Lefèvre, F. Moggia, O. Segut, Y.-P. Lin, Y. Ksari, G. Delafosse, K. Smaali, D. Guérin, V. Derycke, D. Vuillaume, S. Lenfant, L. Patrone and B. Jousselme, J. Phys. Chem. C, 2015, 119, 5703-5713.

42 D. J. Wold and C. D. Frisbie, J. Am. Chem. Soc., 2000, 122, 2970-2971.

43 B. Xu and N. J. Tao, Science, 2003, 301, 1221-1223.

44 S. Guo, J. Hihath, I. Diez-Pérez and N. Tao, J. Am. Chem. Soc., 2011, 133, 19189-19197.

45 I. Bâldea, Nanotechnology, 2014, 25, 455202.

46 As a word of caution, one should mention that the foregoing analysis on the coupling (a)symmetry refers to symmetric molecules (and $\mathrm{C}_{60}$ of ref. 13 is one thereof).

47 A. J. Bard and L. R. Faulkner, Electrochemical methods: fundamentals and applications, Wiley, New York, 1980. 